\title{
Sensor-Assisted Localization in Cellular Systems
}

\author{
Liang Xiao, Student Member, IEEE, Larry J. Greenstein, Life Fellow, IEEE, \\ and Narayan B. Mandayam, Senior Member, IEEE
}

\begin{abstract}
We investigate the use of an auxiliary network of sensors to locate mobiles in a cellular system, based on the received signal strength at the sensor receivers from a mobile's transmission. The investigation uses a generic path loss model incorporating distance effects and spatially correlated shadow fading. We describe four simple localization schemes and show that they all meet E-911 requirements in most environments. Performance can be further improved by implementing the MMSE algorithm, which ideally reaches the Cramer-Rao Bound. We compare the MMSE algorithm and the four simple schemes when the model parameters are estimated via inter-sensor measurements.
\end{abstract}

Index Terms - Cellular systems, localization, mobile terminals, path-loss models, sensor networks.

\section{INTRODUCTION}

$\mathbf{O}$ VER the past few decades, localization technology has been investigated extensively and numerous applications have been proposed in various wireless systems [1]-[8]. In cellular systems, the location information of mobile terminals (MTs) is collected to improve radio resource management, mobility management, and overall cellular system design [1]. Also, the Federal Communications Commission (FCC) initially required all wireless carriers to report the location of E-911 callers with an accuracy of $125 \mathrm{~m}$ in at least $67 \%$ of cases [9]. This rule was later adjusted to $100 \mathrm{~m}$ or less in $67 \%$ of all cases, and $300 \mathrm{~m}$ or less in $90 \%$ of all cases [10].

Here, we investigate the localization of MTs using a network of $N$ spatially dispersed sensors, where the sensors communicate with each other and with the cellular system. A key benefit of this approach is that it provides roundthe-clock measurements from many low-cost devices. Each sensor has an identifying code and a fixed and known location, and it measures the received signal power from transmitting MTs to estimate their locations. Such measurements can also determine the coverage pattern, which can help improve the management of the cellular system [11].

The location of an MT can be determined in various ways [2]. Our work is based on the received signal strength (RSS) or power measurement approach, which is relatively inexpensive and simple to implement in hardware [4]. In the system we consider, it is very likely that some sensors will be located very close to the MT, so that simple-yet-accurate localization schemes should be possible. Simulation results verify this

Manuscript received June 1, 2006; accepted February 22, 2007. The associate editor coordinating the review of this letter and approving it for publication was X. Zhang. This work was supported in part by a grant from Nortel.

The authors are with WINLAB, the Department of Electrical and Computer Engineering, Rutgers University, North Brunswick, NJ, 08902 USA (e-mail: \{1xiao, ljg, narayan\}@winlab.rutgers.edu).

Digital Object Identifier 10.1109/TWC.2007.060316. expectation, as we will show. Specifically, for a cell of radius $1000 \mathrm{~m}, N=200$ sensors (density of 1 sensor per 15,708 $\mathrm{m}^{2}$ ) are sufficient to satisfy the error requirement of E-911.

After a brief overview of related work in Section II, we present the system model in Section III, including a path loss model that incorporates distance effects and spatially correlated shadow fading. We describe the sensor-assisted localization system in Section IV, and present its performance in Section V. In Section VI, we discuss the Cramer-Rao Bound (CRB), the MMSE estimator, and model parameter estimation based on inter-sensor measurements.

\section{RELATED WORK}

Related work in the area of user localization based on received power falls roughly into four categories: (1) Inbuilding infrared networks, (2) cellular networks based on RF, (3) global positioning system (GPS) and (4) sensor networks [3], [5]-[8]. Among them, the MinMax algorithm was proposed for $N$-hop sensor networks to obtain an initial coarse estimate of sensor locations [6]. The least squares (LS) algorithm linearizes the triangular formulas of the distance between anchors (i.e., the sensors with known locations) and the unknown sensor, and then uses the standard least-squares approach to solve the linearized equations [7]. Similarly, in the Euclidean algorithm, up to two possible sensor positions are obtained by strictly solving the triangular formulas of two anchors, and then the position of the unknown sensor is determined by the vote of the third anchor if necessary [8]. It often flags the case with large distance estimation error, through a failure to achieve intersecting circles. Such a flag can signal the location algorithm to switch to a more robust scheme, as we will demonstrate in Section IV.

\section{PATH LOSS Model}

Similar to the case treated in [11], suppose each cell has one MT and $N$ sensors. (We discuss later the case of multiple MTs.) Each of the sensors measures the received power of the uplink pilot signal $P_{i}, i=1, \cdots, N$. We assume that the pilot power measurement is over a bandwidth sufficiently wide (5 $\mathrm{MHz}$ or more) that multipath fading is mostly averaged out. Thus, the measurement of $P_{i}$, combined with knowledge of the uplink pilot transmit power and the antenna gains, permits the network to estimate $P L_{i}$, the path loss between the MT and sensor $i$.

For our purposes, it is safe to assume that the antennas are omni-directional and the pilot powers equal, so that the variation of received power over the sensors precisely tracks the variation of $P L$, i.e., $P_{i}=C-P L_{i}$, where $C$ is the same for (and known by) all sensors. Assuming a generic model 
along the lines of [12], the path loss from the MT to the $i-$ th sensor is

$$
P L_{i}[d B]=A+10 \gamma \log _{10}\left(d_{i} / d_{0}\right)+s_{i} ; \quad i=1, \cdots, N,
$$

where $d_{i}$ is the distance from the MT to sensor $i, s_{i}$ is the shadow fading (in $\mathrm{dB}$ ) along that path, and $d_{0}$ is a reference distance. The intercept $A$ and path loss exponent $\gamma$ are generally unknown and variable from cell to cell. Typically [12], $A$ is close to $20 \log _{10}\left(4 \pi d_{0} / \lambda\right)$, where $\lambda$ is wavelength and $d_{0}$ is specified to be $100 \mathrm{~m}^{1} ; \gamma$ ranges from 3 to $6 ; s_{i}$ is a Gaussian process over space with zero mean and standard deviation $\sigma$; and $\sigma$ is also variable from cell to cell, ranging from $3 \mathrm{~dB}$ to $10 \mathrm{~dB}$ [13]. We assume, for study purposes, that the autocorrelation of the spatial process $s_{i}$ depends only on the separation distance, i.e.,

$$
E\left[s_{i} s_{j}\right]=\sigma^{2} e^{-d_{i j} / X_{c}}
$$

where $d_{i j}$ is the distance from sensor $i$ to sensor $j$; and $X_{c}$, the shadow fading correlation distance, can range from several to many tens of meters [13].

\section{SEnSOR-Assisted Localization System}

Power-based localization in a sensor-assisted cellular system can be implemented in two steps:

Step 1: The system collects the received signal power information $P_{i}$ from $N$ sensors. We denote the sensor locations as $\underline{L}_{1}$, $\cdots, \underline{L}_{i}, \cdots, \underline{L}_{N}$, where $\underline{L}_{i}=\left[x_{i}, y_{i}\right]^{T}, i=1,2, \cdots, N$. The received power and path loss associated with location $L_{i}$ are $P_{i}$ and $P L_{i}$, respectively. For convenience, $i$ is ordered such that $P_{i}$ decreases with $i\left(P_{1} \geq P_{2} \geq \cdots \geq P_{N}\right)$. For most of the schemes considered here, the distances $d_{i}$ is estimated from $P L_{i}$, assuming that the model parameters $A$ and $\gamma$ are known and the shadow-fading components $s_{i}$ are unknown. Estimating $d_{i}$ is possible by assuming that $s_{i}=0$, leading to

$$
\hat{d}_{i}=d_{0} \cdot 10^{\left(P L_{i}-A\right) /(10 \gamma)}
$$

The estimation error for $d_{i}$ results from the existence of unknown shadow fading and any errors in estimating $A$ and $\gamma$.

Step 2: To estimate the location of the MT, $\underline{\theta}=\left[\theta_{1}, \theta_{2}\right]^{T}$, we select the first $n(\in\{1, \cdots, N\})$ data values, i.e., the data collected by sensors with the $n$ strongest powers. Compared with localization algorithms using data from all $N$ sensors [3], this not only simplifies the implementation and saves energy, but also improves the estimate accuracy, as we will discuss in Section V.

The postulated sensor-based system can use existing localization algorithms, such as MinMax [6] and Least Squares [7], but we also consider two new schemes (Weighted Average and Modified Euclidean), as follows:

Weighted Average (WtdAv) Method: This method requires no a priori information on $A$ or $\gamma$, and does not need to estimate the distances between the MT and the sensors. The

\footnotetext{
${ }^{1}$ Since $A$ is common to all path loss terms, we simplify matters, with no loss in generality, by assuming $A=0 \mathrm{~dB}$ throughout this study.
}

location of the MT is assumed to be an average of the locations of the nearby sensors, weighted by their received signal powers, i.e., $\underline{\hat{\theta}}=\left[\sum_{i=1}^{n} x_{i} w_{i}, \sum_{i=1}^{n} y_{i} w_{i}\right]^{T}$, where $w_{i}=P_{i} / \sum_{j=1}^{n} P_{j}$.

Modified Euclidean (ModEuc) Method: This method is an extension of the Euclidean algorithm [8], wherein WtdAv with $n \geq 3$ is invoked if and only if the Euclidean algorithm fails to produce intersecting circles. We will see that ModEuc provides a good combination of accuracy and robustness to conditions; and obviously, it has better coverage than the Euclidean algorithm, especially under heavy shadow fading. For example, with $\gamma=3.8, \sigma_{s}=8 \mathrm{~dB}, X_{c}=80 \mathrm{~m}$, and $N=200$ sensors in an outdoor cell with a radius of 1000 $\mathrm{m}$, the Euclidean algorithm fails in $63 \%$ of the cases where ModEuc succeeds.

Our aim is to examine the four algorithms, MinMax, Least Squares (LS), WtdAv and ModEuc in the context of a network of $N$ power-measuring sensors of known locations. We initially assume that path loss parameters $A$ and $\gamma$ are perfectly known, and we will compare their performances under that condition (Section V). Then we will showcase the minimum mean-square error (MMSE) estimator, which achieves the theoretical lower bound (Cramer Rao Bound (CRB)) under ideal conditions, and compare it with the four simple schemes (VI). In that comparison, we will also account for imperfect knowledge of the model parameters.

\section{Simulations, Results And Discussion}

\section{A. Simulation Approach}

We developed a simulation platform for determining the error statistics for different propagation conditions and system parameters. If not specified otherwise, the numerical results we present will be for the specific case of an outdoor cell, with a cell radius of $1 \mathrm{~km}$; a reference distance $d_{0}=100 \mathrm{~m}$; a frequency of $2.4 \mathrm{GHz}$; a path loss exponent, $\gamma=3.8$; and a correlation distance $X_{c}=80 \mathrm{~m}$.

In the simulations, we first generate a total of $N_{s h}$ "scenarios", a scenario consisting of a randomly chosen MT location and a spatial distribution of the $\mathrm{dB}$ shadow fading component, $s$, as characterized by (2). For each scenario, we generated $M$ random placements of $N$ sensors, with $M$ being a program variable. We chose $N_{s h}=50$ and $M=300$, for a total of 15,000 trials. In each trial, we determined the location estimate for each of the methods, compared it with the true MT location, and thus determined the location error, $\varepsilon$, in meters. From the 15,000 values of $\varepsilon$ for each method, we obtained a cumulative distribution function (CDF), determined the 67th percentile value, and also computed the RMS value.

\section{B. Numerical Results}

As noted, the localization schemes select $n$ out of $N$ data values, i.e., the data collected by those sensors with the $n$ strongest powers. Fig. 1 presents the impact of $n$ on the 67 th percentile and the RMS estimation error, with $3 \leq n \leq 32$ and the parameters indicated in the caption. We see that WtdAv is not sensitive to $n$, because the additional data brought in by a larger $n$ are weighted by smaller values $\left(P_{i} / \sum_{j} P_{j}\right)$. ModEuc is also insensitive to $n$, since the Euclidean method itself is 


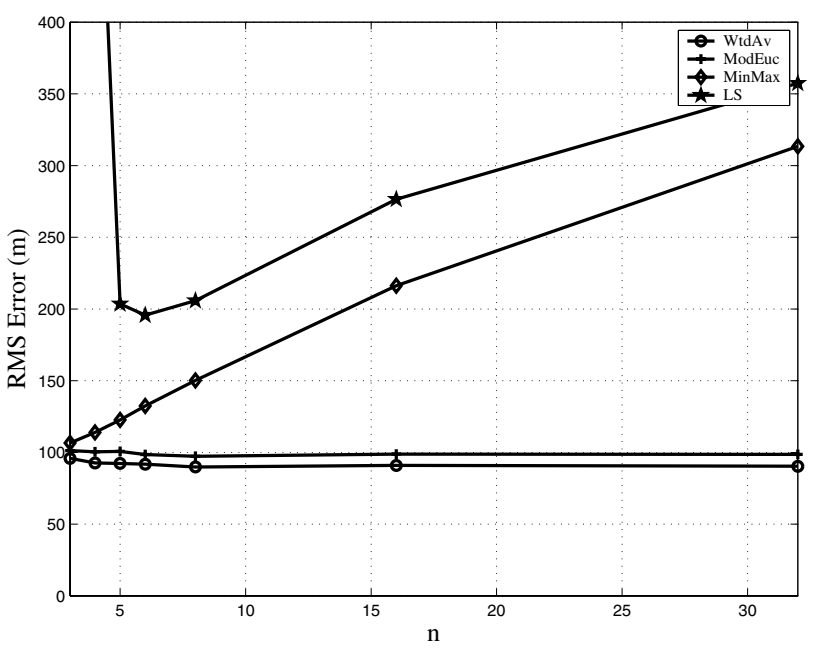

(a) RMS Error (m)

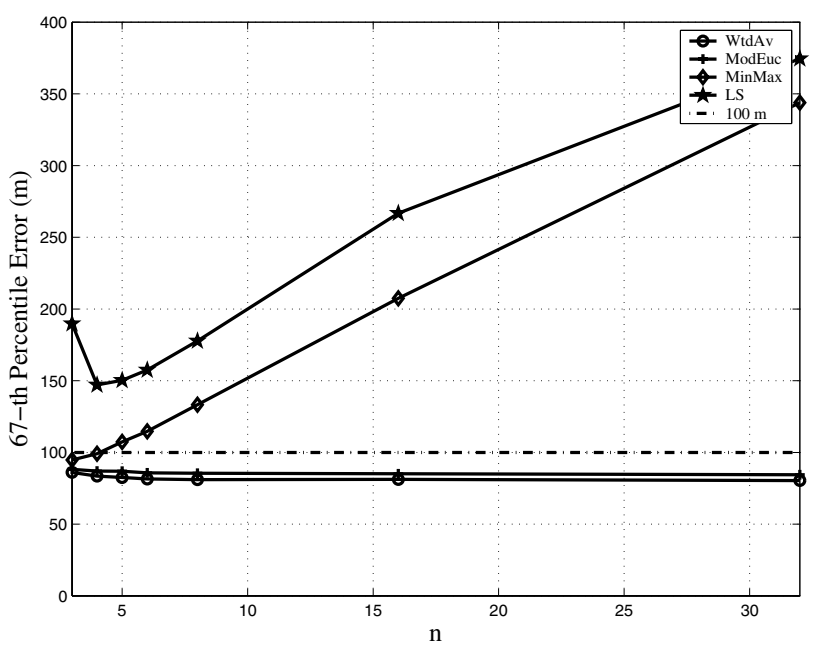

(b) $67 \%$ Error $(\mathrm{m})$

Fig. 1. Error metrics vs. $n$, the number of strongest-power sensors used in the algorithm $\left(\gamma=3.8, \sigma=8 \mathrm{~dB}, X_{c}=80 \mathrm{~m}, N=200\right.$, outdoor cell with a radius of $1000 \mathrm{~m}$ ). Note the rough similarity of the RMS error to the $67 \%$ error in most cases.

based solely on the three nearest sensors; thus, $n>3$ only applies when WtdAv is brought in.

MinMax tends to be less accurate as $n$ rises. From Eq. (1) and Eq. (3) we see that the absolute estimation error of the distance between MT and the $i$-th sensor, $\left|\hat{d}_{i}-d_{i}\right|=$ $d_{i}\left|10^{s_{i} /(10 \gamma)}-1\right|$, is approximately proportional to the distance $d_{i}$ itself. Thus the distance estimate from a farther sensor is usually less accurate, especially in these sparsely distributed sensor networks. Since MinMax does not weight the measurements, its performance degrades when taking into account more "bad" data. Similar comments apply to the LS scheme as well. The choice of $n$ in that case is a bit complicated: $n=4$ and 5 yield the lowest 67 th percentile, while $n=6$ yields the lowest RMS error. The large RMS errors seen for $n<5$ arise from the occasional flip ambiguity (illustrated in [14]), where the sensors line up and cause the estimated and true MT location to be approximately symmetric about the line of sensors. We can assume that a practical algorithm would avoid these cases, but to simplify our study (and since the 67th percentiles are hardly different for $n=4,5$ and 6), we will assume $n=6$ for the LS method.

Now we consider the selection of $N$, the number of sensors in each cell. Fig. 2 shows that the estimation error decreases with $N$ for each scheme. Under large shadow fading $(\sigma=8$ $\mathrm{dB}), \mathrm{LS}$ is the worst algorithm and all other three schemes satisfy, for $N>150$, the FCC requirement that the estimation error be less than $100 \mathrm{~m}$ for $67 \%$ of the cases. For the case with negligible shadow fading $(\sigma=0.1 \mathrm{~dB})$, all schemes satisfy the FCC requirement using very small $N$. Note that the LS and ModEuc schemes yield almost zero error with small shadow fading (assuming $A$ and $\gamma$ are precisely known).

From Fig. 3 we can see that the LS scheme is the most sensitive to $\sigma$ among the four schemes, followed by the ModEuc scheme. However, the latter has smaller 67 th percentile errors than others, as $\sigma$ ranges from 0 to $8 \mathrm{~dB}$. The performance of the WtdAv scheme is similar to that of MinMax, although the former does not require any a priori information about $A$ and $\gamma$. Besides, we can see that $N=200$ is large enough (for a $1-\mathrm{km}$ radius) to find a scheme satisfying the FCC requirement over a wide range of $\sigma$. In an earlier study [11], we found that 200 sensors also enable the system to obtain accurate estimates of outage probability.

Simulation results not presented here show that the estimation error of the algorithms decreases with increasing $\gamma$. For example, the 67th percentile errors of MinMax are 90 $\mathrm{m}$ and $140 \mathrm{~m}$, respectively, for $\gamma=3.8$ and 3, with $\sigma=8$ $\mathrm{dB}, X_{c}=80 \mathrm{~m}$, and $N=200$. This is because the distance estimate is less accurate with a smaller $\gamma$ for the same shadow fading value.

\section{LOWER BOUndS AND PARAMETER Estimation}

To better assess the above results, we first invoke the CRB, which is the theoretical lower limit on the variance of an unbiased estimator. We then describe the MMSE estimator, which meets the CRB if the path loss model parameters are perfectly known; and we show how these parameters can be estimated using the $N$ deployed sensors. Finally, we compare RMS errors corresponding to the CRB, the MMSE estimator and the four simple schemes when the parameter estimates are imperfect. We do this for a situation specifically devised to simplify analysis, i.e., for spatially white shadow fading $\left(X_{c}=0\right)$. To simplify computations as well, we further assume that $N$ and the cell radius are reduced $(N=20$ and the radius is $316 \mathrm{~m}$ ), thereby keeping sensor density the same while significantly reducing the running time.

\section{A. Cramer-Rao Bound (CRB)}

The CRB is the lower bound for the variance of any unbiased estimator, and provides a benchmark for determining how far practical location algorithms are from ideal [15]. Following the derivation in [2], we compute the Fisher information matrix, $\mathbf{F}$, in Eq. (4), for the estimator of $\underline{\theta}$ with observation $\underline{z}=\left[P L_{1}, P L_{2}, \cdots, P L_{N}\right]^{T}$. 


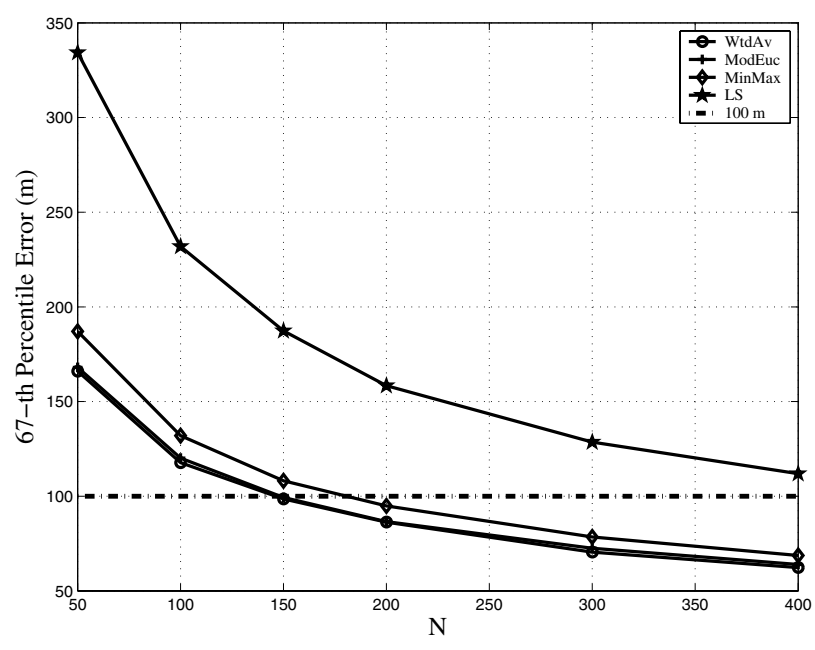

(a) $\sigma=8 \mathrm{~dB}$

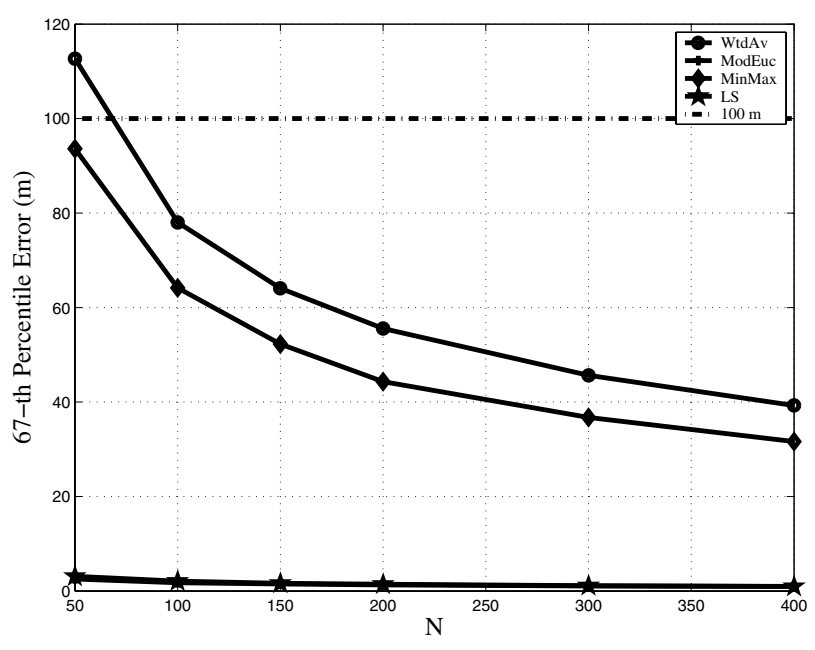

(b) $\sigma=0.1 \mathrm{~dB}$

Fig. 2. $67 \%$ error vs. $N$, the number of sensors in a cell $\left(\gamma=3.8, X_{c}=80 \mathrm{~m}\right.$, outdoor cell with a radius of $\left.1000 \mathrm{~m}\right)$.

$$
\mathbf{F}=\left(\frac{10 \gamma}{\sigma_{s} \log 10}\right)^{2} E_{\underline{\theta}, \underline{L}_{1}, \cdots, \underline{L}_{N}}\left(\left[\begin{array}{cc}
\sum_{i} \frac{\left(\theta_{1}-x_{i}\right)^{2}}{d_{i}^{4}} & \Sigma_{i} \frac{\left(\theta_{1}-x_{i}\right)\left(\theta_{2}-y_{i}\right)}{d_{i}^{4}} \\
\Sigma_{i} \frac{\left(\theta_{1}-x_{i}\right)\left(\theta_{2}-y_{i}\right)}{d_{i}^{4}} & \Sigma_{i} \frac{\left(\theta_{2}-y_{i}\right)^{2}}{d_{i}^{4}}
\end{array}\right]\right)
$$

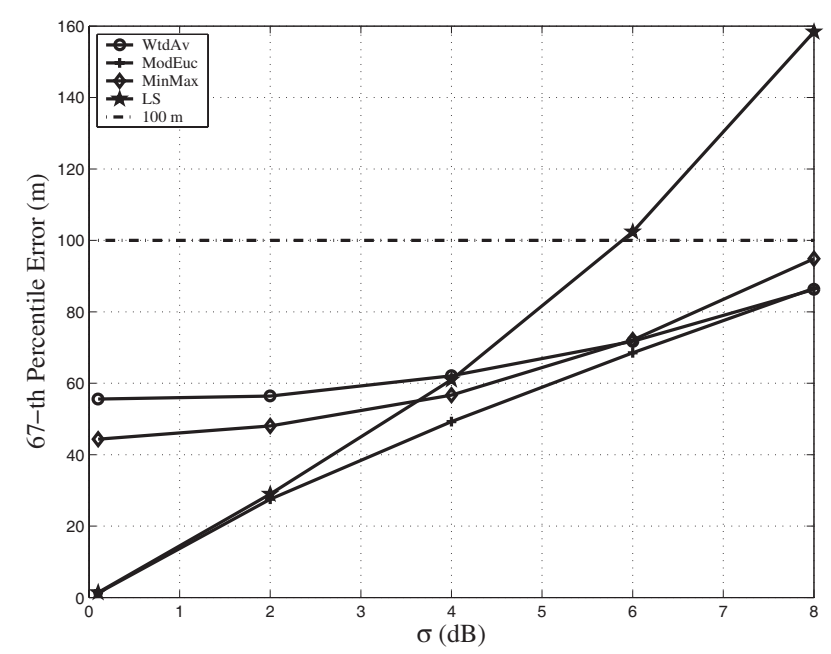

Fig. 3. $67 \%$ error vs. $\sigma\left(N=200, \gamma=3.8, X_{c}=80 \mathrm{~m}\right.$, outdoor cell with a radius of $1000 \mathrm{~m}$ ).

For any unbiased estimator based on power measurements, we have

$$
E\left\{\|\underline{\theta}-\underline{\hat{\theta}}\|^{2}\right\} \geq C R B=\left(\mathbf{F}^{-1}\right)_{1,1}+\left(\mathbf{F}^{-1}\right)_{2,2}
$$

Note that the MT and sensor locations $\left(\underline{\theta}, \underline{L}_{1}, \cdots, \underline{L}_{N}\right)$ are viewed here as random variables, instead of as nonrandom parameters as in [2]. This is because most existing estimators, including the bias-corrected ML estimator in [16], are usually biased for a specific asymmetric MT-sensors topology. Meanwhile, most estimates of $\underline{\theta}$ become unbiased, if averaged over all possible topologies, because of the symmetry. Assuming that the MT and $N$ sensors are uniformly distributed in the cell, we can use (4) to numerically calculate the CRB. If an efficient estimate exists, the CRB can be approached by the
MMSE estimator [15], discussed next.

\section{B. MMSE Estimator}

For the channel model in Section III, the minimum-meansquare error (MMSE) estimate of $\underline{\theta}$ with observations of $\underline{z}$ and known sensor locations is given by Eqs. (6) and (7) (next page), where $p(\underline{\theta})$ has a value equal to the inverse of the cell area if $\underline{\theta}$ is inside the cell, and is zero otherwise. Implementation of the MMSE algorithm clearly requires knowledge of the channel model parameters, $A, \gamma$, and $\sigma$. We now discuss their estimation from finite measurements.

\section{Estimating the Model Parameters}

Among the four schemes studied in Section IV, only WtdAv can operate without knowledge of the model parameters, (1), which are known to vary from cell to cell. The other three rely on knowledge of $A$ and $\gamma$, and Figs. 1-3 are based on perfect information. The MMSE estimator, moreover, requires knowledge of $\sigma$ as well. It also requires an inordinate amount of computation, (6), which is not readily accomplished in a real-time operation like localization. Quantifying this computational problem is beyond the scope of this paper, but we can address the method, and impact, of parameter estimation.

We assume that the $N$ sensors in a cell measure the $N(N-$ 1)/2 path losses among them. The sensor network knows their locations, and thus the distance between each node pair. A scatter plot of path loss vs. distance, with $N(N-1) / 2$ points, can therefore be constructed; and $(A, \gamma, \sigma)$ can be estimated via least-squares fitting using (1). This was done for the case of the smaller cell with 20 sensors and spatially white shadow fading described above, and the results were used to compare RMS errors for various cases. 


$$
\begin{gathered}
\hat{\theta}_{i}=\int_{-\infty}^{\infty} \theta_{i} p\left(\theta_{i} \mid \underline{z}\right) d \theta_{i}=\frac{\int_{-\infty}^{\infty} \int_{-\infty}^{\infty} \theta_{i} p(\underline{z} \mid \underline{\theta}) p(\underline{\theta}) d \theta_{1} d \theta_{2}}{\int_{-\infty}^{\infty} \int_{-\infty}^{\infty} p(\underline{z} \mid \underline{\theta}) p(\underline{\theta}) d \theta_{1} d \theta_{2}}, \quad i=1,2 \\
p(\underline{z} \mid \underline{\theta})=\frac{\exp \left(-\sum_{1 \leq i \leq N}\left(P L_{i}-A+10 \gamma \log _{10}\left(d_{0}\right)-5 \gamma \log _{10}\left(\left(\theta_{1}-x_{i}\right)^{2}+\left(\theta_{2}-y_{i}\right)^{2}\right)\right)^{2} /\left(2 \sigma_{s}^{2}\right)\right)}{\left(\sqrt{2 \pi} \sigma_{s}\right)^{N}}
\end{gathered}
$$

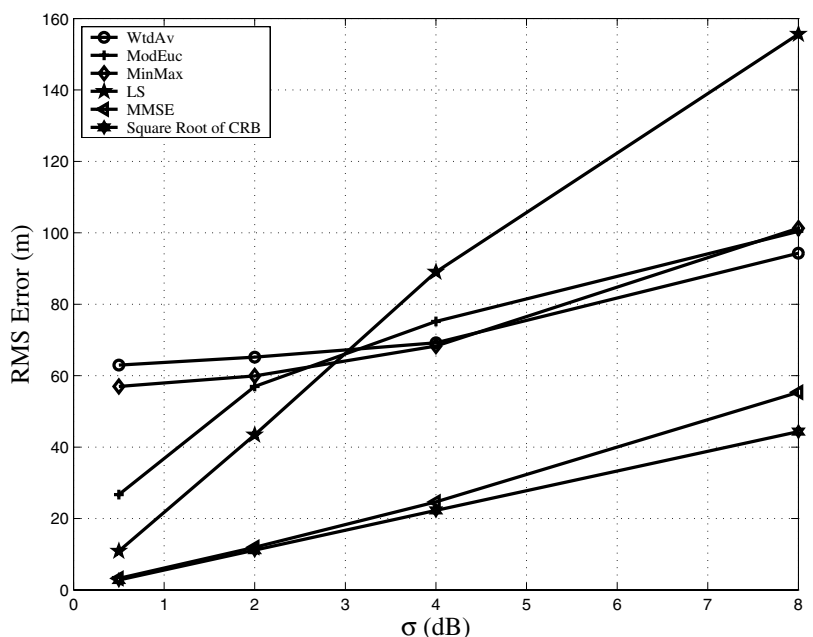

Fig. 4. RMS error vs. $\sigma$, with channel parameters $(A, \gamma$ and $\sigma)$ estimated via least-squares fitting of $N(N-1) / 2$ inter-sensor path loss measurements $\left(N=20, \gamma=3.8, X_{c}=0\right.$, and outdoor cell with a radius of $\left.316 \mathrm{~m}\right)$.

\section{Numerical Results}

Fig. 4 compares RMS location error that include the four simple schemes, the MMSE estimator, and the CRB (actually, the square root of CRB as given by (5)). We see that, with only 20 sensors, the errors in estimating $(A, \gamma, \sigma)$ lead to but a minor degradation in performance. For larger cells with more sensors, the results should be even better. What penalizes the MMSE estimator is its computation-intensive nature.

Comparing the four simple schemes, with each other and with the MMSE estimator, we see that, over the typical range of $\sigma(\sigma>4 \mathrm{~dB})$, there is little difference among WtdAv, MinMax and ModEuc. Also, the RMS errors for these schemes are above that for the MMSE estimator by a factor between 2 and 3. Choosing among approaches then comes down to balancing location accuracy against computation cost (running time, battery energy). The WtdAv scheme seems to provide the best tradeoff while meeting the FCC accuracy requirements under most conditions.

\section{CONCLUSION}

We have postulated a sensor-assisted localization approach for mobile terminals in cellular systems, where the power measurements obtained from $3 \sim 6$ sensors are used to locate the MT. We have validated it by evaluating the performance of five algorithms in the system. Among them, a very simple scheme called WtdAv has performance similar to MinMax, without requiring any channel parameter information. The MMSE estimator that ideally reaches the Cramer-Rao Bound, on the other hand, requires a priori knowledge of all the channel parameters and has prohibitive numerical complexity for real-time operation. Simulation results show that, in an outdoor cell with a radius of $1000 \mathrm{~m}, 200$ sensors are sufficient for all these schemes to meet FCC E-911 requirements in most cases.

Although we have only discussed the localization of a single MT, our approach has great potential to work with multiple MTs. First, the interference between two MTs is small, unless they are too close to each other, because the localization of one MT solely depends on the measurements from the $3 \sim 6$ closest sensors. Moreover, Base Stations can roughly locate the MTs by sectoring, tracking records, etc; and then the sensors need only provide refinements of these localizations. Further effort is needed to more fully explore this approach.

\section{REFERENCES}

[1] J. Caffery and G. Stuber, "Overview of radiolocation in CDMA cellular systems," IEEE Commun. Mag., vol. 35, pp. 38-45, Apr. 1998.

[2] N. Patwari, J. Ash, S. Kyperountas, A. Hero, R. Moses, and N. Correal, "Locating the nodes, cooperative localization in wireless sensor networks," IEEE Signal Processing Mag., pp. 54-69, July 2005.

[3] K. Langendoen and N. Reijers, "Distributed localization in wireless sensor networks: A quantitative comparison," Computer Networks, pp. 499-518, Nov. 2003.

[4] P. Bahl and V. Padmanabhan, "RADAR: An in-building RF-based user location and tracking system," in Proc. IEEE INFORCOM, Mar. 2000, vol. 2, pp. 775-784.

[5] L. Doherty, K. Pister, and L. Ghaoui, "Convex position estimation in wireless sensor networks," in Proc. IEEE INFOCOM, 2001, vol. 3, pp. $1655-1663$.

[6] A. Savvides, H. Park, and M. Srivastava, "The bits and flops of the $N$ hop multilateration primitive for node localization problems," in Proc. 1st ACM International Workshop Wireless Sensor Networks Application, 2002, pp. 112-121.

[7] B. Parkinson and J. Spilker, Global Positioning System: Theory and Application. Washington, DC: American Institute of Astronautics and Aeronautics, 1996.

[8] D. Niculescu and B. Nath, "Ad-hoc positioning system," in Proc. IEEE GLOBECOM, Nov. 2001, vol. 5, pp. 2926-2931.

[9] FCC, "Revision of the commission's rules to ensure compatibility with enhanced 911 emergency calling systems," F. D. N. 94-102, July 1996.

[10] F. NEWS, "FCC adjusts its rules to fasilitate the facilitate the development of nationwide enhanced wireless 911 systems," Sep. 2000.

[11] L. Xiao, L. Greenstein, N. Mandayam, and S. Periyalwar, "Sensor networks for estimating and updating the performance of cellular system," in Proc. IEEE International Conf. Commun., June 2006, vol. 5, pp. $2107-2111$.

[12] V. Erceg, et al., "An empirically based path loss model for wireless channels in suburban environments," IEEE J. Select. Areas Commun., vol. 17, pp. 1205-1211, July 1999.

[13] M. Gudmundson, "Correlation model for shadow fading in mobile radio systems," Electron Lett., vol. 27, pp. 2145-2146, Nov 1991.

[14] D. Moore, J. Leonar, D. Rus, and S. Teller, "Robust distributed network localization with noisy range measurements," in Proc. 2nd ACM SenSys, Nov. 2004, pp. 50-61.

[15] M. Srinath, P. Rajasekaran, and R. Viswanathan, Introduction to Statistical Signal Processing with Applications. Englewood Cliffs, NJ: Prentice Hall, 1996.

[16] N. Patwari and A. Hero, "Using proximity and quantized RSS for sensor localization in wireless networks," in ACM International Conf. Wireless Sensor Networks Applications, 2003, pp. 20-29. 